\title{
Erratum to: Impairment of SOD1-G93A motility is linked to mitochondrial movement in axons of hippocampal neurons
}

Jae Ryul Bae ${ }^{1}$ Sung Hyun Kim ${ }^{1,2}$

Published online: 7 September 2016

(C) The Pharmaceutical Society of Korea 2016

Erratum to: Arch. Pharm. Res. (2016)

39:1144-1150

DOI 10.1007/s12272-016-0798-5

apologize for this confusion or ambiguity caused regarding format of our article.

It is note that this article initially has been published as a review article, however, it is a research article. We here correct this paper as an original research article. We

The online version of the original article can be found under doi:10.1007/s12272-016-0798-5.

Sung Hyun Kim

sunghyunkim@khu.ac.kr

1 Department of Biomedical Science, Graduate School, Kyung Hee University, Seoul 130-701, South Korea

2 Department of Physiology, School of Medicine,

Neurodegeneration Control Research Center, Kyung Hee University, Seoul 130-701, South Korea 